\title{
Urokinase-Type Plasminogen Activator Receptor (uPAR) PET/MRI of Prostate Cancer for Noninvasive Evaluation of Aggressiveness: Comparison with Gleason Score in a Prospective Phase 2 Clinical Trial
}

\author{
Marie Øbro Fosb $\varnothing 1^{1}$, Sorel Kurbegovic ${ }^{1}$, Helle Hjorth Johannesen ${ }^{1}$, Martin Andreas Røder ${ }^{2}$, Adam Espe Hansen ${ }^{1}$, \\ Jann Mortensen ${ }^{1}$, Annika Loft ${ }^{1}$, Peter Meidahl Petersen ${ }^{3}$, Jacob Madsen ${ }^{1}$, Klaus Brasso ${ }^{2}$, and Andreas Kjaer ${ }^{1}$ \\ ${ }^{1}$ Department of Clinical Physiology, Nuclear Medicine \& PET and Cluster for Molecular Imaging, Rigshospitalet and University of \\ Copenhagen, Copenhagen, Denmark; ${ }^{2}$ Copenhagen Prostate Cancer Center, Department of Urology, Rigshospitalet, Copenhagen, \\ Denmark; and ${ }^{3}$ Department of Clinical Oncology, Rigshospitalet, Copenhagen, Denmark
}

The aim of this study was to evaluate the correlation between uptake of the PET ligand ${ }^{68} \mathrm{Ga}-\mathrm{NOTA}-\mathrm{AE} 105$, targeting the urokinase-type plasminogen activator receptor (UPAR), and Gleason score in patients undergoing prostate biopsy. Methods: Patients with clinical suspicion of prostate cancer $(\mathrm{PCa})$ or previously diagnosed with PCa were prospectively enrolled in this phase 2 trial. A combination of UPAR PET and multiparametric MRI (mpMRI) was performed, and the SUV in the primary tumor, as delineated by mpMRI, was measured by 2 independent readers. The correlation between the SUV and the Gleason score obtained by biopsy was assessed. Results: A total of 27 patients had histologically verified $\mathrm{PCa}$ visible on $\mathrm{mpMRI}$ and constituted the study population. There was a positive correlation between the SUV $\max$ and the Gleason score (Spearman $\rho=0.55 ; P=0.003$ ). Receiver operating characteristic analysis showed an area under the curve of $0.88(95 \% \mathrm{Cl}$, 0.67-1.00) for discriminating a Gleason score of greater than or equal to $3+4$ from a Gleason score of less than or equal to $3+$

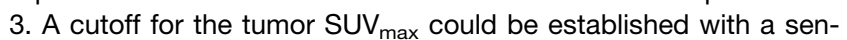
sitivity of $96 \%(79 \%-99 \%)$ and a specificity of $75 \%(30 \%-95 \%)$ for detecting a Gleason score of greater than or equal to $3+4$. For discriminating a Gleason score of greater than or equal to $4+3$ from a Gleason score of less than or equal to $3+4$, a cutoff could be established for detecting a Gleason score of greater than or equal to $4+3$ with a sensitivity of $93 \%(69 \%-99 \%)$ and a specificity of $62 \%$ (36\%-82\%). Conclusion: SUV measurements from uPAR PET in primary tumors, as delineated by $\mathrm{mpMRI}$, showed a significant correlation with the Gleason score, and the tumor SUV $\max$ was able to discriminate between low-risk Gleason score profiles and intermediate risk Gleason score profiles with a high diagnostic accuracy. Consequently, uPAR PET/MRI could be a promising method for the noninvasive evaluation of $\mathrm{PCa}$ and might reduce the need for repeated biopsies (e.g., in active surveillance).

\footnotetext{
Received Apr. 29, 2020; revision accepted Jun. 30, 2020.

For correspondence or reprints contact: Andreas Kjaer, Department of Clinical Physiology, Nuclear Medicine \& PET, Rigshospitalet, Blegdamsvej 9, DK 2100 Copenhagen, Denmark.

E-mail: akjaer@sund.ku.dk

Published online Aug. 6, 2020.

COPYRIGHT @ 2021 by the Society of Nuclear Medicine and Molecular Imaging.
}

Key Words: urokinase-type plasminogen activator receptor; prostate cancer; PET/MRI; risk stratification; active surveillance; Gleason score

J Nucl Med 2021; 62:354-359

DOI: $10.2967 /$ jnumed.120.248120

$\mathbf{P}$ rostate cancer $(\mathrm{PCa})$ is highly variable in nature, ranging from a low-risk disease that can be monitored by active surveillance to a more aggressive disease that requires prompt definitive treatment. Accurate methods for risk assessment are important not only for reducing the number of unnecessary interventions in patients with low-risk disease but also for avoiding delays in potential curative therapy in patients with higher risk disease (1-3). Current stratification is based on serum prostate-specific antigen (PSA) level, clinical tumor stage, and histopathologic grading of biopsy material with the Gleason score (GS). Recently, multiparametric MRI (mpMRI) was incorporated into PCa guidelines for detection and as a tool for targeting biopsies (4,5). Using mpMRI for targeting biopsies increased the number of clinically significant cancers detected-an important advancement in the management of PCa (5-7).

During active surveillance, repeated biopsies to detect histopathologic progression are key elements. However, repeated biopsies may show results different from those of the primary biopsy as a consequence of sampling error. To circumvent sampling error and to replace an invasive method with a noninvasive method, molecular imaging targeting markers of aggressiveness (e.g., PET) could be ideal. The risk of sampling error in tissue biopsies is overcome, as the entire tumor volume is assessed by imaging. In this context, combining PET and mpMRI is an appealing option, in which mpMRI serves the goal of achieving a reproducible delineation of the tumor to guide the PET readout.

The urokinase-type plasminogen activator receptor (uPAR) is a cell membrane protein involved in extracellular matrix degradation. In addition to regulating proteolysis, uPAR activates many intracellular signaling pathways that promote invasion and proliferation through cooperation with transmembrane receptors. uPAR is frequently overexpressed in malignant tissue, and high 
expression levels are associated with cancer invasion, metastatic potential, and resistance to chemotherapy (8-12). In clinical studies of prostatectomy specimens, uPAR expression levels in tumor tissue were associated with a higher pathologic tumor stage, higher GS, positive surgical margins, and shorter biochemical recurrence-free survival $(13,14)$. These observations support the notion that noninvasive evaluation of UPAR expression could become a clinically relevant prognostic imaging biomarker, with the possibility of distinguishing indolent tumors from the invasive phenotype; this approach subsequently could be used as a noninvasive replacement for biopsies during active surveillance.

For UPAR PET, several radioligands based on the high-affinity peptide antagonist AE105 have been developed (15-19). Phase 1 studies with both ${ }^{68} \mathrm{Ga}-\mathrm{NOTA}-\mathrm{AE} 105$ and ${ }^{64} \mathrm{Cu}$-DOTA-AE105 in patients with various cancer types, including $\mathrm{PCa}$, have been published $(20,21)$. Both ligands accumulated in primary tumor lesions as well as metastases, and the uptake corresponded with high UPAR expression in excised tumor tissue; these data provided evidence for uPAR PET imaging being target specific.

The aim of this exploratory phase 2 study was to investigate the use of ${ }^{68} \mathrm{Ga}$-NOTA-AE105 uPAR PET as a tool for the evaluation of localized PCa. Accordingly, the predefined primary outcome measure was the study of the correlation between UPAR ligand uptake in the primary tumor and GS obtained from biopsy material.

\section{MATERIALS AND METHODS}

\section{Study Design}

Between November 2017 and September 2019, we included 52 men fulfilling 1 of 2 criteria: either suggestion of PCa on the basis of clinical findings or previously diagnosed PCa before repeat biopsies in an active surveillance program. Included patients underwent uPAR $\mathrm{PET} / \mathrm{mpMRI}$ before the initiation of any antitumor therapy. Exclusion criteria were obesity (body weight of $>140 \mathrm{~kg}$ because of scanner safety limits), severe claustrophobia, or metallic foreign bodies not compatible with MRI. Eligible patients were included after giving informed consent.

The PCa diagnosis was verified histologically after transrectal MRI-directed cognitive fusion-guided biopsy. GS and localization of lesions from the pathology report served as references for the study outcome.

The study protocol was approved by the Danish Health and Medicine Authority (EudraCT no. 2017-002276-37; sponsor Rigshospitalet) and the Ethical Committee of the Capital Region of Denmark (protocol no. $\mathrm{H}-17019311$ ). Written informed consent was obtained from all patients. The study was registered at ClinicalTrials.gov (NCT03307460) and was performed in accordance with the recommendations for good clinical practice, including independent monitoring by the Good Clinical Practice Unit of the Capital Region of Denmark.

\section{PET/mpMRI Acquisition}

${ }^{68} \mathrm{Ga}$-NOTA-AE105 PET/mpMRI was performed using an integrated whole-body PET/MRI system (Siemens Biograph mMR; Siemens Healthcare). PET was performed as a 45-min scan starting at the injection of approximately $200 \mathrm{MBq}$ of ${ }^{68} \mathrm{Ga}-\mathrm{NOTA}-\mathrm{AE} 105$. Synthesis of the ligand was performed as previously described (21). Patients were asked to void before the examination; no bladder catheter or rectal coil was applied. Patients crossed their arms over their chest, and their arms did not enter the field of view at the level of the prostate.

PET data were reconstructed using vendor-provided Dixon-based MR attenuation correction with atlas-based bone segmentation and absolute scatter correction (3-dimensional ordinary Poisson-ordered-subset expectation maximization [3D-OP-OSEM], 3 iterations, 21 subsets, 4$\mathrm{mm}$ gaussian filter). Images used for interpretation and quantification were from the reconstruction of data acquired 20-30 min after the injection of ${ }^{68} \mathrm{Ga}-\mathrm{NOTA}-\mathrm{AE} 105$.

\section{mpMRI Protocol}

The 3-T MRI parameters were as follows: transverse T1-weighted turbo spin-echo (TSE) (echo time [TE], $20 \mathrm{~ms}$; repetition time [TR], $735 \mathrm{~ms}$; pixel size, $0.7 \times 0.7 \mathrm{~mm}$; slice thickness, $5.0 \mathrm{~mm}$ ); vendorprovided Dixon-volumetric interpolated breath-hold examination for PET attenuation correction (voxel size, $1.3 \times 1.3 \times 3.0 \mathrm{~mm}$ ); sagittal T2-weighted TSE (TE, $101 \mathrm{~ms}$; TR, 5,590 ms; pixel size, $0.6 \times$ $0.6 \mathrm{~mm}$; slice thickness, $3.0 \mathrm{~mm}$ ); coronal T2-weighted TSE (TE, $101 \mathrm{~ms}$; TR, 6,050 ms; pixel size, $0.6 \times 0.6 \mathrm{~mm}$; slice thickness, $3.0 \mathrm{~mm}$ ); transverse T2-weighted TSE (TE, $104 \mathrm{~ms}$; TR, 7,480 ms; pixel size, $0.5 \times 0.5 \mathrm{~mm}$; slice thickness, $3.0 \mathrm{~mm}$ ); diffusion-weighted single-shot spin-echo echoplanar imaging (TE, $60 \mathrm{~ms}$; TR, 5,700 ms; pixel size, $2.0 \times 2.0 \mathrm{~mm}$; slice thickness, $3.0 \mathrm{~mm}$; b values, 0 and 800 $\mathrm{s} / \mathrm{mm}^{2}$; and TE, $66 \mathrm{~ms}$; TR, 5,700 ms; pixel size, $2.0 \times 2.0 \mathrm{~mm}$; slice

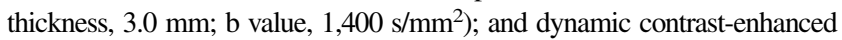
volumetric interpolated breath-hold examination (TE, $1.78 \mathrm{~ms}$; TR, $5.18 \mathrm{~ms}$; pixel size, $1.4 \times 1.4 \times 3.6 \mathrm{~mm}$; 35 repetitions; acquisition time, 4 min $25 \mathrm{~s}$; bolus of gadobutrol (Gadavist; Bayer HealthCare LLC) contrast agent $[0.1 \mathrm{~mL} / \mathrm{kg} ; 1 \mathrm{mmol} / \mathrm{mL}]$ administered after the second repetition).

\section{Image Analysis}

All image data were analyzed by 2 certified specialists in nuclear medicine and an experienced certified specialist in MRI radiology. mpMRI data were interpreted according to Prostate Imaging Reporting and Data System 2.0 (PI-RADS) (22) and reviewed by the radiologist and treating urologist together before mpMRI-directed cognitive fusion-guided biopsy was performed.

Volumes of interest (VOIs) corresponding to lesions identified on mpMRI were drawn on T2-weighted MR images using Mirada DBx (Mirada Medical) without knowledge of the PET results. When necessary, the VOIs were subsequently adjusted by the nuclear medicine specialists to avoid the inclusion of a signal from the urinary bladder. Uptake of the uPAR ligand in the MRI VOIs was parameterized as $\mathrm{SUV}_{\text {max }}$.

\section{Statistical Analysis}

Patient characteristics were reported by descriptive statistics, including age, PSA level, clinical tumor (cT) stage, and pathologic tumor (pT) stage (when available). In case of multiple lesions per patient, the lesion with the highest $\mathrm{SUV}_{\max }$ was included in the analysis. The Spearman rank correlation was used to estimate the correlation between the SUV and pathologic features measured on an ordinal scale. The interreader reliability of SUV measurements was estimated using the ICC. A comparison of the distributions of continuous data was performed with an independent sample $t$ test. Determination of the optimal cutoff for the discrimination of a GS of less than or equal to $3+3$ from a GS of greater than or equal to $3+4$ and of a GS of less than or equal to $3+4$ from a GS of greater than or equal to $4+3$ was performed with an R-package developed by Budczies et al. (23).

A $P$ value of less than 0.05 was considered statistically significant. Statistical analyses were performed using IBM SPSS Statistics v. 22 (IBM Corp.) and R (http://www.R-project.org).

\section{RESULTS}

A total of 52 patients were enrolled in the study. Because of exclusions and technical issues, 46 patients had both PET/MRI and histology available. Of these, 16 patients had no suspect lesions on mpMRI and a further 3 patients had negative histology, leaving a study population of 27 patients for analysis (Fig. 1). Of these, 6 patients were enrolled before repeat biopsies were obtained in an active surveillance program, whereas the remaining 21 patients were 


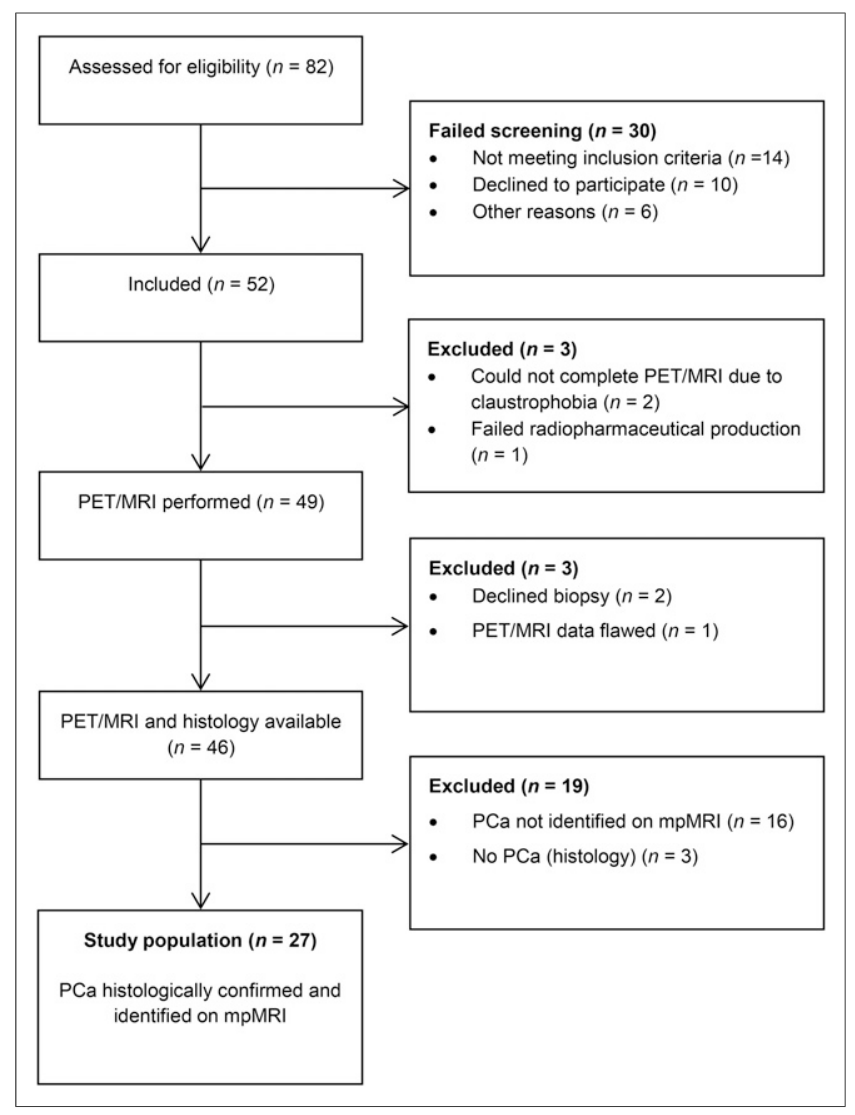

FIGURE 1. Consolidated Standards of Reporting Trials (CONSORT) flow diagram of inclusion process.

enrolled as they were scheduled to undergo biopsies on the basis of clinical suspicion of PCa. Patient characteristics are shown in Table 1.

Patients received an intravenous dose of approximately 200 $\mathrm{MBq}$ (median, $211 \mathrm{MBq}$; range, $154-234 \mathrm{MBq}$ ) of ${ }^{68} \mathrm{Ga}-\mathrm{NOTA}-$ AE105; according to dosimetry calculations from the phase 1 trial, this dose corresponds to an effective dose of approximately 3.1 $\mathrm{mSv}(21)$. None of the patients experienced reactions or adverse events related to the administration.

Interobserver reliability in the measurement of the tumor $\mathrm{SUV}_{\max }$ was excellent, with an ICC of 0.90 (95\% CI, 0.79-0.95). There was a significant positive correlation between the tumor VOI SUV $\mathrm{Sax}_{\max }$ and the GS from biopsy material, with a correlation coefficient of $0.55(P=$ 0.003) (Fig. 2). Table 2 shows the mean $\mathrm{SUV}_{\max }$ based on the GS.

To estimate the ability of the $\mathrm{SUV}_{\max }$ to discriminate between GS groups, receiver operating characteristic analyses were performed. For discrimination between a GS of less than or equal to $3+3$ and a GS of greater than or equal to $3+4$, the area under the curve for the $\mathrm{SUV}_{\max }$ was 0.88 (95\% CI, 0.67-1.00) $(P=$ 0.017). The optimal cutoff for achieving greater than $90 \%$ sensitivity for detecting a GS of greater than or equal to $3+4$ was an $\mathrm{SUV}_{\max }$ of 2.75 (Fig. 3). The suggested cutoff showed a sensitivity of $95.7 \%(79.0 \%-99.2 \%)$, a specificity of $75 \%$ (30.1\%-95.4\%), an accuracy of $88.0 \%(67.7 \%-100.0 \%)$, and an odds ratio of 7.5 (1.16-48.63) $(P=0.005)$ for the detection of a GS of greater than or equal to $3+4$. Likewise, if a sensitivity of greater than $90 \%$ for the detection of a GS of greater than or equal to $4+3$ were wanted, for example, to identify high-risk PCa during uPAR
TABLE 1

Characteristics of 27 Patients

\begin{tabular}{|c|c|c|c|c|}
\hline Characteristic & Mean & Range & $\begin{array}{l}\text { No. of } \\
\text { patients }\end{array}$ & $\begin{array}{c}\% \text { of } \\
\text { patients }\end{array}$ \\
\hline Age $(y)$ & 66.6 & $50.2-79.9$ & & \\
\hline $\begin{array}{l}\text { PSA level } \\
(\mathrm{ng} / \mathrm{mL})\end{array}$ & 28.8 & $7.0-56.0$ & & \\
\hline \multicolumn{5}{|l|}{$\begin{array}{l}\text { Clinical tumor } \\
\text { stage }\end{array}$} \\
\hline cT1 & & & 9 & 33.3 \\
\hline сT2 & & & 9 & 33.3 \\
\hline сT3 & & & 9 & 33.3 \\
\hline \multicolumn{5}{|l|}{ GS } \\
\hline $3+3$ & & & 4 & 14.8 \\
\hline $3+4$ & & & 9 & 33.3 \\
\hline $4+3$ & & & 5 & 18.5 \\
\hline $\begin{array}{l}8(4+4 / 3 \\
\quad+5 / 5+3)\end{array}$ & & & 2 & 7.4 \\
\hline $\begin{array}{l}9(4+5 / 5 \\
+4)\end{array}$ & & & 7 & 25.9 \\
\hline \multicolumn{5}{|l|}{$\begin{array}{l}\text { PI-RADS } \\
\text { score }\end{array}$} \\
\hline 4 & & & 10 & 37.0 \\
\hline 5 & & & 17 & 63.0 \\
\hline
\end{tabular}

image-based active surveillance, then a cutoff of an $\mathrm{SUV}_{\max }$ of 3.725 could be applied (Fig. 4). For discrimination of a GS of greater than or equal to $4+3$ from a GS of less than or equal to $3+4$, the suggested cutoff resulted in a sensitivity of $92.9 \%(68.5 \%-98.7 \%)$, a specificity of $61.5 \%(35.5 \%-82.3 \%)$, an accuracy of $75.8 \%(56.7 \%-$ $95.0 \%)$, and an odds ratio of $2.44(1.08-5.53)(P=0.004)$. Examples of $\mathrm{SUV}_{\max }$ readouts in 2 patients with a GS of 8 and a GS of $3+4$ are shown in Figure 5.

Seventeen patients underwent radical prostatectomy; 3 (17.6\%) were upgraded in GS compared with their biopsy results (2 patients with a GS of $3+3$ and 1 patient with a GS of $4+3$ ),

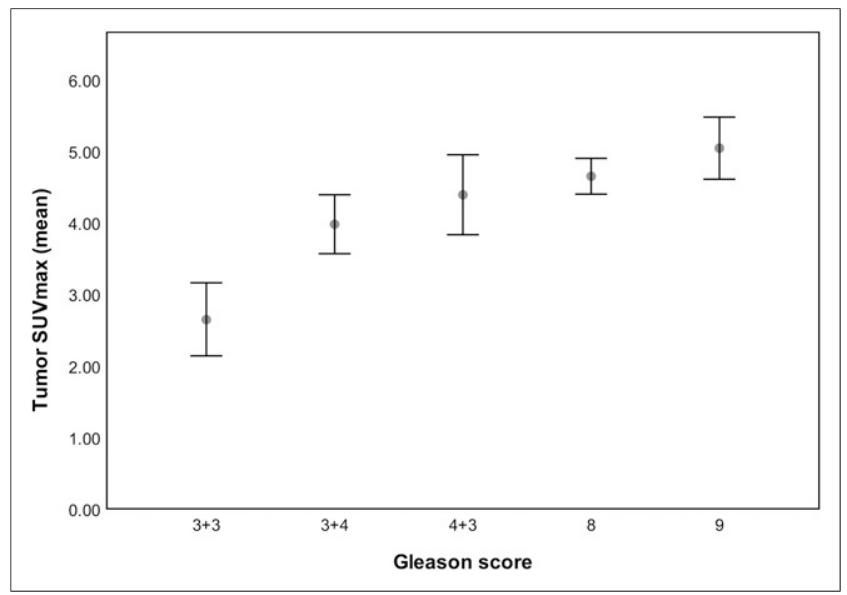

FIGURE 2. Relationship between UPAR PET SUV $\max$ and GS. Values are reported as mean and SEM. 
TABLE 2

SUV $_{\max }$ for GS Subgroups

\begin{tabular}{lcccc}
\hline GS & $\begin{array}{c}\text { No. of } \\
\text { patients }\end{array}$ & $\begin{array}{c}\text { Reader 1 } \\
\text { mean SUV }\end{array}$ & $\begin{array}{c}\text { Reader 2 } \\
\text { mean SUV }\end{array}$ & $\begin{array}{c}\text { Mean } \\
\text { SUV }_{\max }\end{array}$ \\
\hline $3+3$ & 4 & $2.48(0.54)$ & $2.83(0.53)$ & $2.65(0.51)$ \\
$3+4$ & 9 & $4.13(0.46)$ & $3.82(0.38)$ & $3.98(0.41)$ \\
$4+3$ & 5 & $4.80(0.91)$ & $3.98(0.22)$ & $4.39(0.56)$ \\
8 & 2 & $4.65(0.25)$ & $4.65(0.25)$ & $4.65(0.25)$ \\
9 & 7 & $5.22(0.49)$ & $4.86(0.41)$ & $5.04(0.43)$
\end{tabular}

Values in parentheses are SEMs.

whereas 2 patients (11.7\%) were downgraded ( 1 with a GS of $4+4$ and 1 with a GS of $4+5$ ). Applying the suggested $S_{U V}$ max cutoff for discriminating a GS of greater than or equal to $4+3$ from a GS of less than or equal to $3+4$ resulted in a sensitivity of $88.9 \%$ $(51.8 \%-99.7 \%)$, a specificity of $62.5 \%(24.5 \%-91.5 \%)$, and an accuracy of $76.5 \%(50.1 \%-93.2 \%)$. As only 1 patient had a GS of $3+3$ in final histopathology, the suggested $S_{U V} V_{\max }$ cutoff for identifying a GS of greater than or equal to $3+4$ has not been tested in this subpopulation.

There was no significant correlation between the $\mathrm{SUV}_{\max }$ and the PSA level or between the GS and the PSA level.

\section{DISCUSSION}

The main finding and primary outcome measure of this phase 2 clinical trial using the uPAR-targeted PET ligand ${ }^{68} \mathrm{Ga}-\mathrm{NOTA}-$ AE105 in PCa patients were a correlation between uPAR PET ligand uptake and the GS obtained from biopsy material. The data

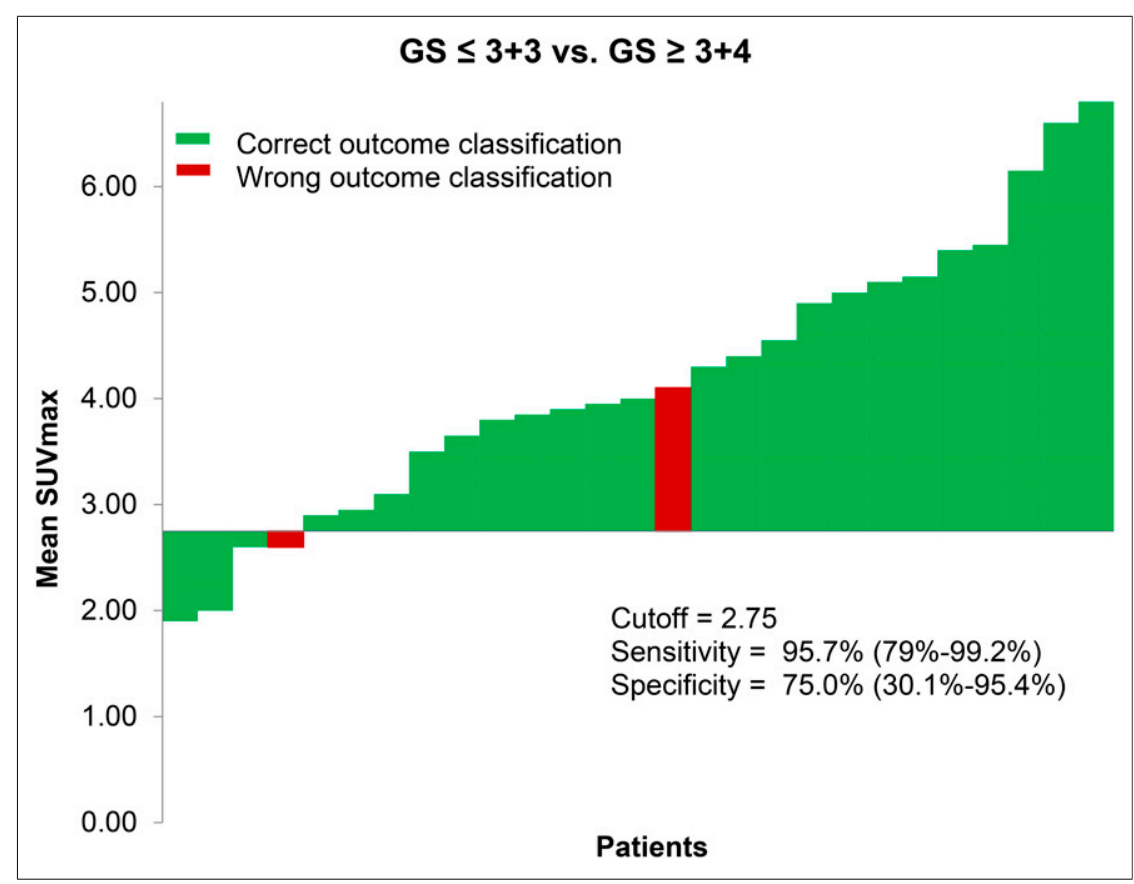

FIGURE 3. Waterfall plot of SUV $V_{\max }$ in tumors for discrimination of GS of $\leq 3+3$ from GS of $\geq 3+4$. suggest that UPAR PET could serve as a substitute for at least some invasive biopsies. An $\mathrm{SUV}_{\max }$ cutoff with a high sensitivity for discriminating patients with a GS of greater than or equal to $3+4$ from patients with a GS of less than or equal to $3+3$ could be established and could provide important prognostic information regarding adverse surgical pathology, risk of biochemical recurrence, metastasis, and PCa-specific mortality (24). Especially for patients in active surveillance, this method may be valuable for noninvasively evaluating the need for repeated biopsies. Furthermore, the addition of UPAR PET to mpMRI could be applied in biopsy guidance, when indicated, to target lesions with the highest $\mathrm{SUV}_{\max }$ and therefore the highest uPAR expression.

uPAR PET readouts should be obtained in the tumor is why imagefusion with MRI or other tumor-delineating imaging is needed. Tumoral ligand uptake, especially in tumors with a low GS, is not sufficient for delineation of a tumor on PET images alone. as the strength of uPAR PET is the dynamic range, leading to low uptake in low-grade tumors. Also, and for the same reason, UPAR PET is unlikely to be useful for metastatic staging, for which other modalities should be applied.

PET ligands targeted to prostate-specific membrane antigen (PSMA) have also been investigated for the noninvasive evaluation of the GS. Some clinical studies of PSMA PET found a correlation between ${ }^{68} \mathrm{Ga}$-PSMA-11 uptake and the GS, mainly driven by high uptake in tumors with a GS of 9 or 10 (25-27). However, the published results showed considerable overlap among all GS categories, making the establishment of meaningful cutoff values impossible. Furthermore, a PET/MRI study with prostatectomy histopathology as a reference found no correlation between ${ }^{68} \mathrm{Ga}$-PSMA-11 uptake and the GS (28). Although PSMA PET undoubtedly can play a major role in the management of PCa patients, for example, in staging, it cannot be considered an appropriate method for GS estimation.

One might speculate whether the correlation with GS obtained by UPAR PET could have been obtained through mpMRI alone. Our study was not designed to address this question, and we cannot rule out that mpMRI in these patients would have correlated with GS. However, if this is the case, we only see this as an advantage, in particular, if the combined information obtained from uPAR PET and mpMRI could lead to an even better correlation with the GS. Therefore, in future large-scale studies, we plan to use a design that can compare the information obtained from UPAR PET with that obtained from mpMRI as well as to study the added value of combining the 2 modalities and whether doing so could lead to an even stronger correlation between imaging and GS.

As an exploratory, single-center phase 2 clinical trial, the present study had inherent limitations. Because of the limited number of observations, the statistical analysis and diagnostic performance had broad CIs. However, the results of the present study will serve as an encouraging basis for designing future phase 3 clinical trials with larger numbers of patients. Further studies are needed before more firm conclusions 


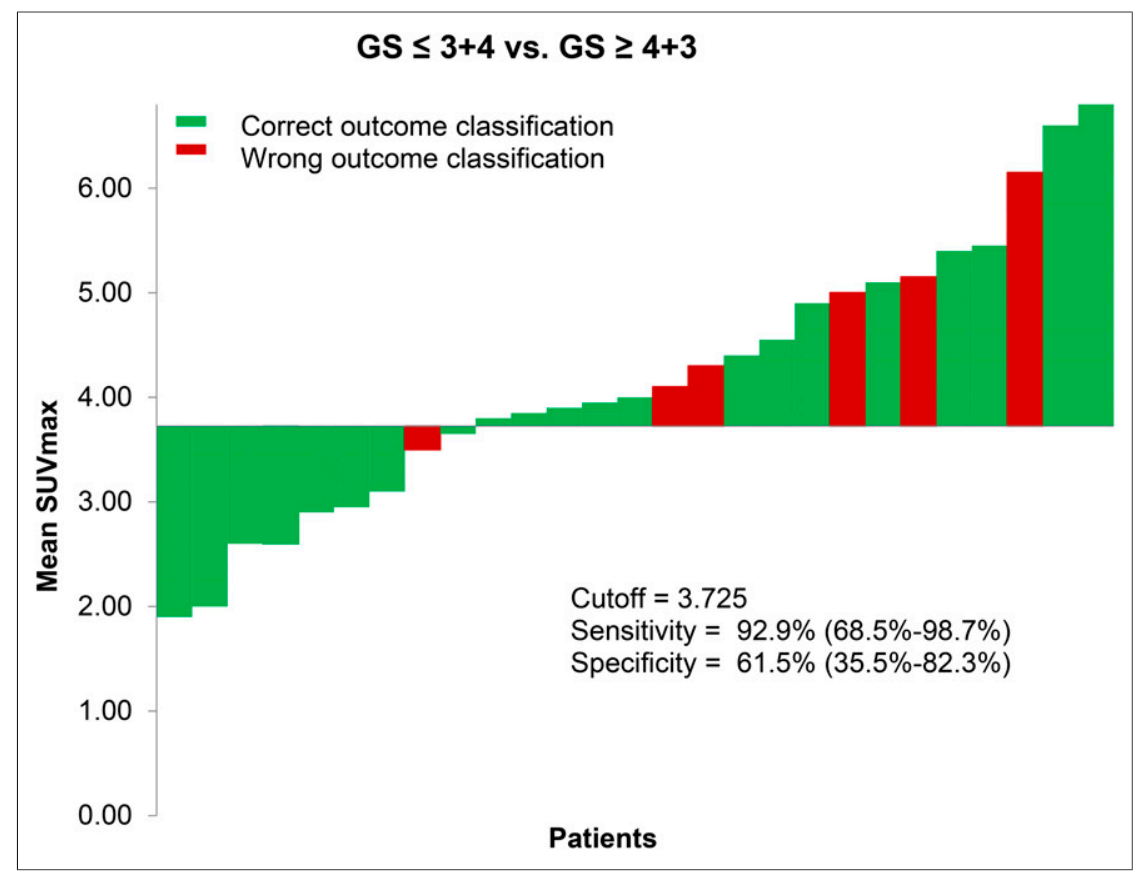

FIGURE 4. Waterfall plot of $S U V_{\max }$ in tumors for discrimination of $G S$ of $\leq 3+4$ from $G S$ of $\geq 4+3$.

can be drawn about the clinical utility and potential of UPAR PET/ mpMRI. Furthermore, when based on much larger numbers of patients, the SUV $\mathrm{max}_{\text {max }}$ cutoff values can be more firmly established and the added value of integrated PET/mpMRI can be investigated.

Another issue that needs to be addressed is the selection of biopsies as a reference for the PET/mpMRI results. Biopsies are prone to sampling error, as documented by relatively frequent upor downgrading of the GS in studies of prostatectomy specimens $(29,30)$. Biopsies were chosen as the primary reference in the present study because our aim was to investigate whether uPAR PET could serve as a noninvasive alternative to the current biopsy regimen. Our study included patients with low-risk disease on active surveillance - a population of particular interest in the context of noninvasive risk stratification and for whom the utility of uPAR PET may be highest. However, prostatectomy is not routinely performed in such patients; therefore, biopsies are the only accessible samples for histologic grading in these patients. Reducing the number of biopsies in active surveillance would be of significant

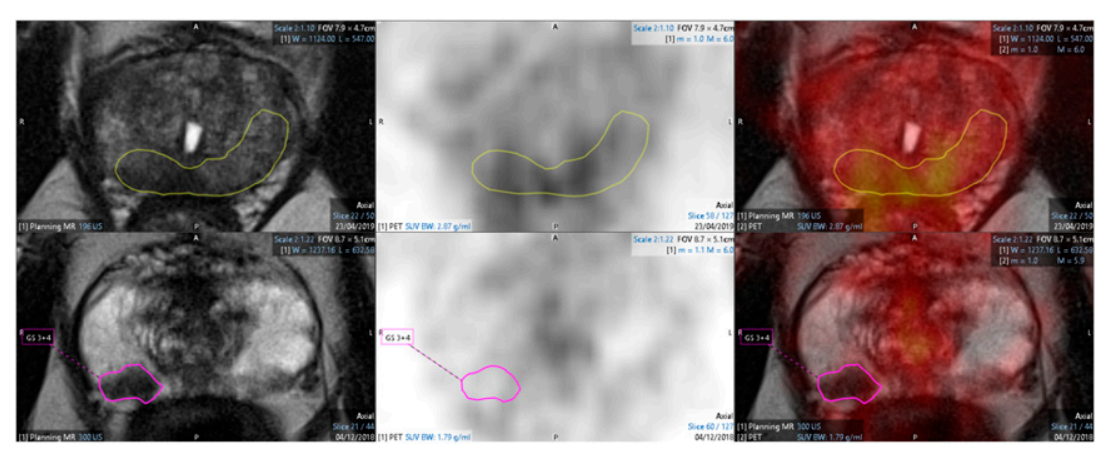

FIGURE 5. Delineated tumor VOIs in UPAR PET/MRI (transaxial T2-weighted images) of patient who had PCa with GS of 8 (top; SUV $\max =4.6$ ) and patient who had PCa with GS of $3+4$ (bottom; SUV $\max =2.8$ ). value, as biopsies not only are uncomfortable for the patient but also are hampered by side effects such as bleeding and infection (31).

In our study population, a subgroup did undergo prostatectomy; of these patients, nearly $30 \%$ had a different GS on final pathology compared with the previous biopsy. Although based on a limited number of patients, data from prostatectomies also supported our results regarding the SUV cutoff for the discrimination of a GS of greater than or equal to $4+3$ from a GS of less than or equal to $3+4$.

Another limitation of the present study was the selection of index lesions. We decided to include only lesions with a PIRADS score of greater than or equal to 4 to reduce the number of false-positive results. As most patients who have lesions with a PI-RADS score of 4 or 5 have clinically significant $\mathrm{PCa}$, it could be argued that the study population did not adequately represent patients with low-risk disease. However, a substantial number of the patients included in the present study were candidates for active surveillance. Especially in these patients, uPAR PET may aid in determining the need for close monitoring. A future study should specifically be aimed at patients in active surveillance and should include lower-grade PI-RADS lesions.

As we used MRI-guided uPAR PET, ideally the biopsies in the study cohort should also have been obtained by MRI-targeted fusion biopsy (software-assisted MRI-transrectal ultrasound fusion or inbore MRI target biopsy). However, at the time of inclusion of the patients, the equipment for software-assisted fusion or in-bore biopsy was not available at our facility, prompting the application of MRIdirected cognitive registration. This method has been reported to yield lower rates of detection of PCa compared with other MRI targeting techniques $(32,33)$. However, we expect that such biopsies most likely would correlate even better with our uPAR PET imaging biopsies.

\section{CONCLUSION}

This phase 2 clinical trial of uPAR PET/MRI using ${ }^{68} \mathrm{Ga}$-NOTAAE105 showed promising results for use in PCa patients. uPAR PET ligand accumulation correlated with the GS, indicating that the noninvasive evaluation of PCa might replace at least some biopsies in active surveillance. On the basis of current data, a phase 3 study is under planning to further investigate the utility of this approach in active surveillance. In addition, studies in various clinical settings of PCa are also planned to investigate the independent prognostic value of UPAR PET.

\section{DISCLOSURE}

This project received funding from the European Union's Horizon 2020 Research and Innovation Programme under grant agreements 670261 (ERC Advanced Grant) 
and 668532 (Click-It), the Lundbeck Foundation, the Novo Nordisk Foundation, the Innovation Fund Denmark, the Danish Cancer Society, the Arvid Nilsson Foundation, the Svend Andersen Foundation, the Neye Foundation, the Research Foundation of Rigshospitalet, the Danish National Research Foundation (grant 126), the Research Council of the Capital Region of Denmark, the Danish Health Authority, the John and Birthe Meyer Foundation, and the Research Council for Independent Research. Andreas Kjaer and Jacob Madsen are inventors on a patent of the composition of matter of UPAR PET (WO 2014086364) and are cofounders of Curasight, which holds IPR for the UPAR PET technology. Curasight offered to support the study economically. However, as sufficient funding was raised from other sources, no economic support from Curasight was provided. No other potential conflict of interest relevant to this article was reported.

\section{KEY POINTS}

QUESTION: Is there a correlation between uptake of the PET ligand ${ }^{68} \mathrm{Ga}-\mathrm{NOTA}-\mathrm{AE} 105$, targeting the UPAR, and the GS in PCa?

PERTINENT FINDINGS: A phase 2 clinical trial of UPAR PET/MRI in 27 patients with $\mathrm{PCa}$ found that the SUV $\mathrm{max}_{\text {in tumors had a }}$ significant positive correlation with the GS obtained by biopsy. SUV thresholds could be established to discriminate between a low-risk GS and an intermediate-risk GS.

IMPLICATIONS FOR PATIENT CARE: UPAR PET/MRI could be a promising method for the noninvasive evaluation of $\mathrm{PCa}$ and might reduce the need for repeated biopsies (e.g., in active surveillance).

\section{REFERENCES}

1. Tosoian JJ, Mamawala M, Epstein JI, et al. Intermediate and longer-term outcomes from a prospective active-surveillance program for favorable-risk prostate cancer. J Clin Oncol. 2015;33:3379-3385.

2. Thurtle D, Rossi SH, Berry B, Pharoah P, Gnanapragasam VJ. Models predicting survival to guide treatment decision-making in newly diagnosed primary nonmetastatic prostate cancer: a systematic review. BMJ Open. 2019;9:e029149.

3. Gnanapragasam VJ, Bratt O, Muir K, et al. The Cambridge Prognostic Groups for improved prediction of disease mortality at diagnosis in primary non-metastatic prostate cancer: a validation study. BMC Med. 2018;16:31.

4. Mottet N, van den Bergh RCN, Briers E, et al. EAU prostate cancer guidelines. Edn. presented at the EAU Annual Congress; 2019; Barcelona, Spain. https:// uroweb.org/guideline/prostate-cancer/. Accessed December 15,2020.

5. Drost FH, Osses DF, Nieboer D, et al. Prostate MRI, with or without MRItargeted biopsy, and systematic biopsy for detecting prostate cancer. Cochrane Database Syst Rev. 2019;(4):CD012663.

6. Ahmed HU, El-Shater Bosaily A, Brown LC, et al. Diagnostic accuracy of multiparametric MRI and TRUS biopsy in prostate cancer (PROMIS): a paired validating confirmatory study. Lancet. 2017;389:815-822.

7. Sonn GA, Fan RE, Ghanouni P, et al. Prostate magnetic resonance imaging interpretation varies substantially across radiologists. Eur Urol Focus. 2019;5:592-599.

8. Huang Z, Wang L, Wang Y, et al. Overexpression of CD147 contributes to the chemoresistance of head and neck squamous cell carcinoma cells. J Oral Pathol Med. 2013;42:541-546.

9. Wang K, Xing Z-H, Jiang Q-W, et al. Targeting uPAR by CRISPR/Cas9 system attenuates cancer malignancy and multidrug resistance. Front Oncol. 2019;9:80.

10. Eastman BM, Jo M, Webb DL, Takimoto S, Gonias SL. A transformation in the mechanism by which the urokinase receptor signals provides a selection advantage for estrogen receptor-expressing breast cancer cells in the absence of estrogen. Cell Signal. 2012;24:1847-1855.

11. Rabbani SA, Ateeq B, Arakelian A, et al. An anti-urokinase plasminogen activator receptor antibody (ATN-658) blocks prostate cancer invasion, migration, growth, and experimental skeletal metastasis in vitro and in vivo. Neoplasia. 2010;12:778-788.
12. Mahmood N, Mihalcioiu C, Rabbani SA. Multifaceted role of the urokinase-type plasminogen activator (uPA) and its receptor (uPAR): diagnostic, prognostic, and therapeutic applications. Front Oncol. 2018;8:24.

13. Kumano M, Miyake H, Muramaki M, Furukawa J, Takenaka A, Fujisawa M. Expression of urokinase-type plasminogen activator system in prostate cancer: correlation with clinicopathological outcomes in patients undergoing radical prostatectomy. Urol Oncol. 2009;27:180-186.

14. Cozzi PJ, Wang J, Delprado W, et al. Evaluation of urokinase plasminogen activator and its receptor in different grades of human prostate cancer. Hum Pathol. 2006;37:1442-1451.

15. Persson M, Madsen J, Østergaard S, et al. Quantitative PET of human urokinasetype plasminogen activator receptor with ${ }^{64} \mathrm{Cu}$-DOTA-AE105: implications for visualizing cancer invasion. $\mathrm{J} \mathrm{Nucl} \mathrm{Med.} \mathrm{2012;53:138-145.}$

16. Persson M, Hosseini M, Madsen J, et al. Improved PET imaging of uPAR expression using new ${ }^{64} \mathrm{Cu}$-labeled cross-bridged peptide ligands: comparative in vitro and in vivo studies. Theranostics. 2013;3:618-632.

17. Persson M, Liu H, Madsen J, Cheng Z, Kjaer A. First ${ }^{18}$ F-labeled ligand for PET imaging of uPAR: in vivo studies in human prostate cancer xenografts. Nucl Med Biol. 2013;40:618-624.

18. Persson M, El Ali HH, Binderup T, et al. Dosimetry of ${ }^{64} \mathrm{Cu}-\mathrm{DOTA}-\mathrm{AE} 105$, a PET tracer for uPAR imaging. Nucl Med Biol. 2014;41:290-295.

19. Persson M, Kjaer A. Urokinase-type plasminogen activator receptor (UPAR) as a promising new imaging target: potential clinical applications. Clin Physiol Funct Imaging. 2013;33:329-337.

20. Persson M, Skovgaard D, Brandt-Larsen M, et al. First-in-human uPAR PET: imaging of cancer aggressiveness. Theranostics. 2015;5:1303-1316.

21. Skovgaard D, Persson M, Brandt-Larsen M, et al. Safety, dosimetry, and tumor detection ability of ${ }^{68} \mathrm{Ga}-\mathrm{NOTA}-\mathrm{AE} 105$ : first-in-human study of a novel radioligand for uPAR PET imaging. J Nucl Med. 2017;58:379-386.

22. Vargas HA, Hötker AM, Goldman DA, et al. Updated Prostate Imaging Reporting and Data System (PIRADS v2) recommendations for the detection of clinically significant prostate cancer using multiparametric MRI: critical evaluation using whole-mount pathology as standard of reference. Eur Radiol. 2016;26:1606-1612.

23. Budczies J, Klauschen F, Sinn BV, et al. Cutoff Finder: a comprehensive and straightforward web application enabling rapid biomarker cutoff optimization. PLoS One. 2012;7:e51862.

24. Kane CJ, Eggener SE, Shindel AW, Andriole GL. Variability in outcomes for patients with intermediate-risk prostate cancer (Gleason score 7, International Society of Urological Pathology Gleason group 2-3) and implications for risk stratification: a systematic review. Eur Urol Focus. 2017;3:487-497.

25. Uprimny C, Kroiss AS, Decristoforo C, et al. ${ }^{68} \mathrm{Ga}$-PSMA-11 PET/CT in primary staging of prostate cancer: PSA and Gleason score predict the intensity of tracer accumulation in the primary tumour. Eur J Nucl Med Mol Imaging. 2017;44:941-949.

26. Sathekge M, Lengana T, Maes A, et al. ${ }^{68} \mathrm{Ga}-\mathrm{PSMA}-11 \mathrm{PET} / \mathrm{CT}$ in primary staging of prostate carcinoma: preliminary results on differences between black and white South-Africans. Eur J Nucl Med Mol Imaging. 2018;45:226-234.

27. Hicks RM, Simko JP, Westphalen AC, et al. Diagnostic accuracy of $\left({ }^{68} \mathrm{Ga}\right)$ PSMA-11 PET/MRI compared with multiparametric MRI in the detection of prostate cancer. Radiology. 2018;289:730-737.

28. Eiber M, Weirich G, Holzapfel K, et al. Simultaneous ${ }^{68}$ Ga-PSMA HBED-CC PET/ MRI improves the localization of primary prostate cancer. Eur Urol. 2016;70:829-836.

29. Epstein JI, Feng Z, Trock BJ, Pierorazio PM. Upgrading and downgrading of prostate cancer from biopsy to radical prostatectomy: incidence and predictive factors using the modified Gleason grading system and factoring in tertiary grades. Eur Urol. 2012;61:1019-1024.

30. Treurniet KM, Trudel D, Sykes J, Evans AJ, Finelli A, Van der Kwast TH. Downgrading of biopsy based Gleason score in prostatectomy specimens. J Clin Pathol. 2014;67:313-318.

31. Loeb S, Carter HB, Berndt SI, Ricker W, Schaeffer EM. Complications after prostate biopsy: data from SEER-Medicare. J Urol. 2011;186:1830-1834.

32. Wegelin O, van Melick HHE, Hooft L, et al. Comparing three different techniques for magnetic resonance imaging-targeted prostate biopsies: a systematic review of in-bore versus magnetic resonance imaging-transrectal ultrasound fusion versus cognitive registration-is there a preferred technique? Eur Urol. 2017;71:517-531.

33. Stabile A, Dell'Oglio P, Gandaglia G, et al. Not all multiparametric magnetic resonance imaging-targeted biopsies are equal: the impact of the type of approach and operator expertise on the detection of clinically significant prostate cancer. Eur Urol Oncol. 2018;1:120-128. 